\title{
Patient's perspective during surgical and/or diagnostic procedures performed in day surgery in breast cancer patient. Psychological implications and care pathway quality assessment with a specific questionnaire and a quality score
}

\author{
Marco Cascella ${ }^{*}$, Maurizio Del Giudice ${ }^{1}$, Maria Rosaria Muzio ${ }^{2}$, Francesco Portinaio ${ }^{1}$, Daniela Viscardi ${ }^{3}$, Massimiliano D'Aiuto ${ }^{4}$, Giuseppe \\ Laurelli $^{5}$, Stefano Greggi ${ }^{5}$ and Arturo Cuomo ${ }^{1}$ \\ ${ }^{1}$ Division of Anesthesia, Department of Anesthesia, Endoscopy and Cardiology, Istituto Nazionale Tumori "Fondazione G. Pascale"-IRCSS, Naples, Italy \\ ${ }^{2}$ Division of Infantile Neuropsychiatry, UOMI-Maternal and Infant Health, Asl NA 3 SUD, Torre del Greco, via Marconi, 66 - 80059 Torre del Greco (NA), Italy. \\ ${ }^{3}$ Department of Surgery, Anesthesia, Emergency and Intensive Care - University of Naples "Federico II", Naples, Italy \\ ${ }^{4}$ Department of Breast Oncology, Istituto Nazionale Tumori "Fondazione G. Pascale”-IRCSS, Naples, Italy \\ ${ }^{5}$ Department of Gynecology Oncology, Istituto Nazionale Tumori “Fondazione G. Pascale”-IRCSS, Naples, Italy
}

\begin{abstract}
For much interventions and diagnostic procedures Day Surgery is the ideal therapeutic approach from the point of view of care effectiveness and patient satisfaction. Recently, special attention has been paid to the psychological aspects related to surgical and diagnostic procedures performed in Day Surgery, especially in cancer patient.

In this work we evaluated the efficacy of a questionnaire investigating the entire care pathway of women undergoing surgery or diagnostic procedures in Day Surgery for diagnosed or suspected malignant breast disease. In the questionnaire formulation particular interest has been gave to the doctor-patient relationship psychological aspects and to the surgical procedure elements strongly influencing patient satisfaction and having the greatest impact in perioperative anxiety. The care pathway quality assessment has been completed by the proposal of a specific quality score.
\end{abstract}

\section{Introduction}

For much interventions and diagnostic procedures Day Surgery (DS) is the ideal therapeutic approach from the point of view of care effectiveness and patient satisfaction [1]. As a consequence, a gradual increase of the procedures applied in DS has been observed. This has made important the establishment and the use of guidelines [2], especially for patient selection criteria [3-5].

The aim of DS is the provision of surgical and diagnostic services with reduced costs and time, and contemporary maintaining (or even improving) the quality levels of the services themselves. Recently, moreover, special attention has been paid to the psychological aspects related to procedures performed in DS [6]. The advantages are evident: for example, the certainty of the immediate discharge home reduces worry and anxiety related to hospitalization. However, in DS procedures, the relationships established between patient and medical staff are different from those established in standard hospitalization; in the latter, in fact, the longer hospitalization time naturally allows a gradual familiarization between patients and medical staff, which is hardly established in DS. In DS, hence, a key role is played by the staff for assistant quality of humanity and ability in the establishment of a rapid empathy level [7].
The emotional components are even more relevant in cancer patient or in patients subjected to the surgical and diagnostic procedures aimed at confirmation of a suspected neoplasm [8]. In fact, in addition to the concern related to the intervention, these patients live this experience in a state of great anxiety for the fear of a positive neoplasm diagnosis [9]. These DS related emotional components seem to be even more relevant in women breast cancer cases [10].

When cancer diagnosis procedures are performed in DS, the day of surgery patients experience a profound sense of disorientation due to the fact that an unknown scary event has to be faced. Waiting for the diagnosis and the fear that it will be positive create a state of anxiety and depression associated with panic and stress, due to the feeling of

Correspondence to: Marco Cascella, Division of Anesthesia, Department of Anesthesia, Endoscopy and Cardiology, Istituto Nazionale Tumori "Fondazione G. Pascale”-IRCSS, Naples, Italy, Tel: + 39081 5903586; Fax: + 39081 5903778; E-mail: m.cascella@istitutotumori.na.it

Key words: day surgery, patient satisfaction, breast cancer patient, questionnaire, quality score

Received: March 06, 2015; Accepted: March 24, 2015; Published: March 26, 2015 
Cascella M (2015) Patient's perspective during surgical and/or diagnostic procedures performed in day surgery in breast cancer patient. Psychological implications and care pathway quality assessment with a specific questionnaire and a quality score

disruption of their life projects. The patient, in this condition, have the impression to live in another body, suddenly and dramatically perceived as stranger and possessed by a dangerous disease. This condition persists until the end of the wait, the moment in which the patient is aware of the truth, whatever it is.

In light of these considerations and in order to optimize the quality of provided services, it is necessary to obtain a high degree of patient satisfaction (PS). The main problem in this, is finding the most relevant elements of the care pathway affecting PS. This is especially true in particularly fragile class of patients, e.g. women undergoing surgical and/or diagnostic procedures for malignant breast disease. In these cases, the most important role is played by gathering information on PS, which not only refers to the medical and nursing quality (e.g. ability of operators), but to the whole patient experience during care pathway [11].

The investigation methods used to bring out the users opinion can be direct or indirect. The first one refers to all methods directly involving users, who are asked to express their level of satisfaction on a particular service. Indirect methods instead, allow to know users opinions through the analysis of secondary data or derivatives (e.g. complaints, suggestions, sentinel events, observation etc.). The use of this last method has several disadvantages: I) the analysis of data is a complex process and requires long times; II) large amount of data can be lost and III) they are affected by the difficulty of pondering the impact of the procedure outcome on the patient's psychological state and hence on the quality of judgment. In fact, a diagnostic test resulting in a negative response for neoplasm may predispose the patient to express a favorable judgment on the service provided, and vice versa.

Direct methods like self-assessment questionnaires, semistructured interviews and questionnaires sent by mail are instead to be preferred. These methods offer the possibility to make comparisons both diachronic (the same service during the time) and synchronic (comparison between different services), involving a high number of people.

In this work we formulate a questionnaire investigating the entire care pathway in DS. Particular attention will be given to the doctor-patient relationship, psychological aspects and to the surgical procedure elements (empirically derived from patients reports) strongly influencing PS (e.g. waiting times) and having the greatest impact in perioperative patient anxiety.

\section{Methods}

\section{Study population}

A population of 624 patients from June 2011 to June 2013 has been studied. All patients signed a consent form authorizing the collection of data. We informed patients that there would have been no changes in both surgical and anesthetic techniques commonly used for the management of these interventions.

For the evaluation of the offered services quality, we considered women undergoing surgery or diagnostic procedures in DS at the Breast Surgery Center in the National Cancer Institute of Naples. The types of operations are shown in Table. 1.

\section{The questionnaire}

A self-assessment questionnaire was used by the DS of the National Cancer Institute of Naples (Table 2). It was composed of nine multiple choice questions with three possible answers for each question. The
Table 1. Surgical and diagnostic procedures performed in day surgery in breast cancer patient.

\begin{tabular}{|l|}
\hline Interventions \\
\hline Breast lesions enucleation/enucleore section \\
\hline Lumpectomy (Wide local excision) \\
\hline Central duct resection \\
\hline Diagnostic procedures \\
\hline Ultrasound-guided biopsy \\
\hline
\end{tabular}

Table 2. Self assessment questionnaire.

\begin{tabular}{|c|c|c|c|c|}
\hline 1. & $\begin{array}{l}\text { How do you judge the access to the } \\
\text { Hospital? }\end{array}$ & Easy & Indifferent & Difficult \\
\hline 2. & $\begin{array}{l}\text { How do you judge the waiting time } \\
\text { for surgery (waiting list time)? }\end{array}$ & Short & Acceptable & Long \\
\hline 3. & $\begin{array}{l}\text { How do you judge the waiting time in } \\
\text { the day of surgery? }\end{array}$ & Short & Acceptable & Long \\
\hline & $\begin{array}{l}\text { How do you judge the relationship } \\
\text { established with medical staff? }\end{array}$ & Good & Acceptable & Difficult \\
\hline 5. & $\begin{array}{l}\text { How do you assessment the provided } \\
\text { information level during the entire } \\
\text { care pathway? }\end{array}$ & Good & Acceptable & Insufficient \\
\hline 6. & $\begin{array}{l}\text { How do you judge the medical staff } \\
\text { empathy? }\end{array}$ & Good & Acceptable & Insufficient \\
\hline 7. & $\begin{array}{l}\text { How do you judge the postoperative } \\
\text { care? }\end{array}$ & Good & Acceptable & Inadequate \\
\hline 8. & $\begin{array}{l}\text { How would you assessment the } \\
\text { information level at the discharge? }\end{array}$ & Good & Acceptable & Insufficient \\
\hline & $\begin{array}{l}\text { Would you suggest National Cancer } \\
\text { Institute of Naples to another cancer } \\
\text { patient? }\end{array}$ & Yes & $\begin{array}{l}\text { I don't } \\
\text { know }\end{array}$ & No \\
\hline
\end{tabular}

tenth question of the questionnaire was an open question asking the patient to give advices and express criticism or praise about the received service.

The questionnaire was part of an informative package together with a section devoted to the collection of patient demographics, including the telephone number, meant to be possibly used to contact back the patient for clarifications, explanations or for statistical purposes. The compilation of this sensitive information was optional.

In the questionnaire there was also a section dedicated to the telephone numbers and to all indispensable information useful for the users to withdraw the results of diagnostic tests. The patients at the end of questionnaire compilation, pick this information section off and mail the questionnaire.

The questionnaire was composed of items related to the following topics: healthcare system quality judgment (hotel service, hospitality and comfort); health care settings (with particular attention to the process of information both before and after surgery) and the courtesy and the empathy degree of all personnel involved (medical, nursing, and administrative support). An item was devoted to the waiting time, intended as the time between the booking of the intervention and the date of the procedure completion (waiting list time). Another item, strongly influencing PS, was referred to the waiting time in the day of surgery (waiting between procedures of the acceptance and the intervention completion). The ninth item was conceived as an overall indicator of quality asking the patient if he/she would recommend the health facility just experienced for the future. 
The questionnaire included a final open question in which the patient could indicate any suggestions for improving care. The recognition of these data is essential to keep refining the assistance on the basis of comments and assessments, introducing changes eventually leading to the fulfillment of the patients demand. For the subsequent analysis of data, we defined "unusual responses" all data hardly classifiable (e.g. absence of answer or multiple answers to a given item).

\section{Results}

The DS of the National Cancer Institute of Naples questionnaire discussed in methods section above, allowed us to find and highlight the most common issues in the majority of patients. Moreover it showed how the structure and timing of questions influence the percentage of "unusual responses" obtained.

The first question asked an opinion about the possibility of the Institute admission, intended as the set of procedures for the reservation. The $84 \%$ (n.519) of respondents judged the admission easy, but the $8 \%$ (n.50) of patients found it rather difficult (Graph 1). It is worth saying that probably, for the majority of people interviewed, the question was not properly understood. Many people in fact intended the question as referred to the "physical" access to the Institute (transportation, parking, etc.) as emerged from the negative comments detected in the last open question of the questionnaire ("I had difficulty to park ","I risked being late for a strike of the public transport company" "I was stacked in a traffic jam").

The second question was about the evaluation of the waiting times for surgery (from booking to the day of surgery) (Graph 2). The 52\%

\section{How do you judge the access to the Hospital?}

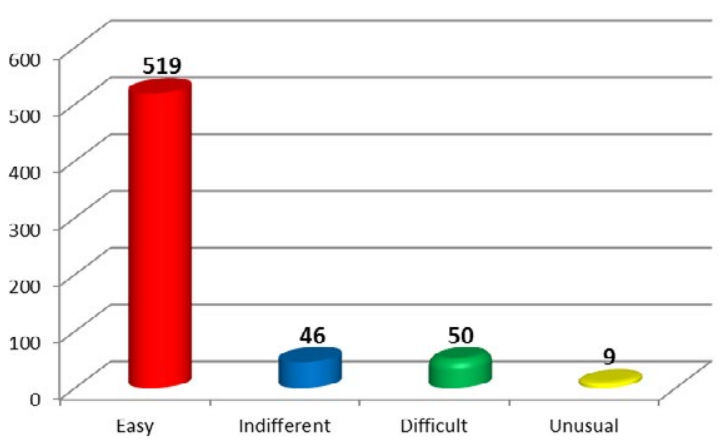

- Easy

Indifferent

Difficult

uUnusual

Graph 1. Question 1.

\section{How do you judge the waiting time for surgery (waiting list time)?}

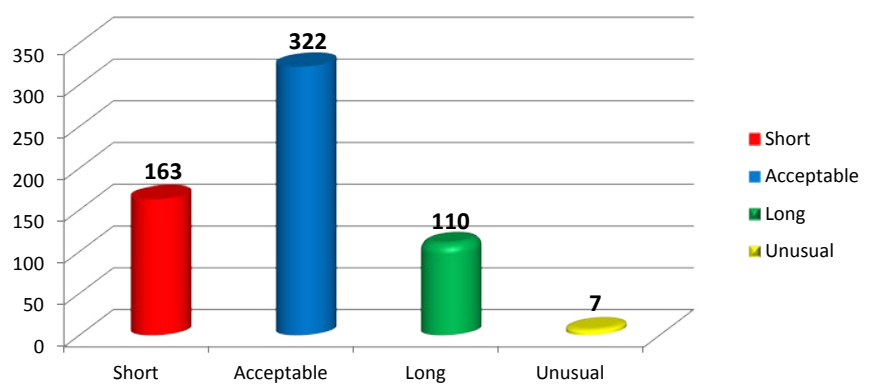

Graph 2. Question 2.

\section{How do you judge the waiting time in the day of surgery?}

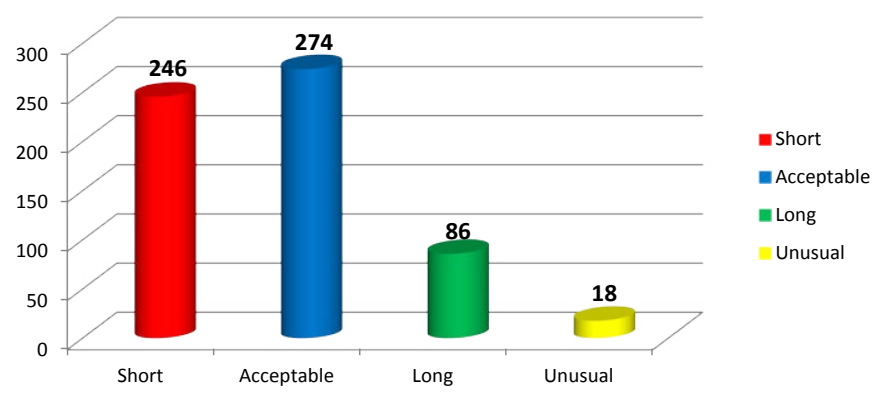

Graph 3. Question 3.

\section{How do judge the relationship established with medical the staff ?}

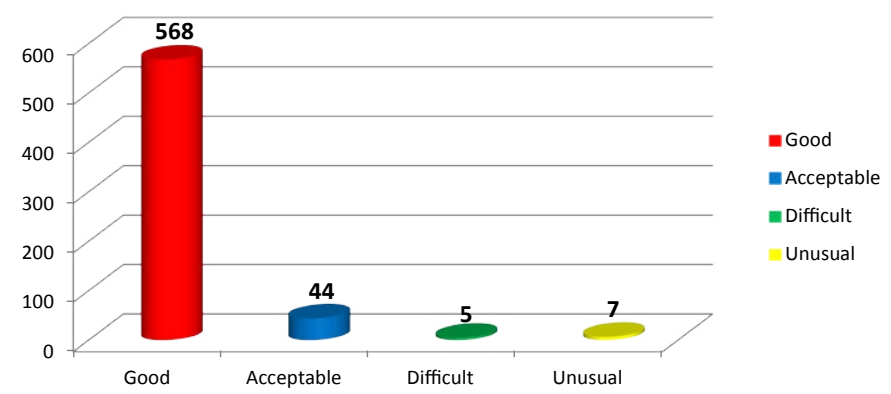

Graph 4. Question 4.

(n.322) of the judged sample found the time spent awaiting surgery acceptable. The $18 \%$ (n.110) considered the wait too long and the $20 \%$ (n. 163) considered it short.

These data highlight the need to shorten as much as possible the waiting time, operating drastic interventions for the reduction of the waiting lists length.

The waiting time in the day of surgery (waiting between procedures of acceptance and intervention completion) had a great impact in anxiety (Graph 3).The 39.5\% (n.246) of the judged sample considered the waiting time in the surgery morning short; the $44 \%$ (n.274) found it acceptable; the $14 \%$ (n.86) considered it too long, and the 3\% (n.18) gave an "unusual response".

Many people intended "the waiting time" as referred to the discharge wait from the hospital in the surgery day. Patients wished to leave the hospital as soon as possible, they didn't understand the required time to the discharge and the postoperative recovery time.

The fourth question, evaluating the relationship between patients and medical team established in the Operating Theatre, judged the care quality (Graph 4). The 91\% (n.568) of the judged sample established a good relationship. The 7\% (n.44) considered it acceptable, and only the $1 \%$ (n.5) found it difficult.

The percentages showed how the patients appreciated the staff ability to give psychological support in the Complex Operative and how this ability influenced the PS.

The fifth question asked a judgment about the overall provided 
information level during the entire care pathway (Graph 5). The obtained results were quite positive: the $72 \%$ (n.447) of the judged sample considered the provided information level good; the $23 \%$ (n.146) judged it acceptable, and the $4 \%$ (n.25) considered it insufficient. As emerged from the comments detected in the last open question of the questionnaire, the patients judged the information received in the day of surgery good, but they considered the information provided before admission (e.g. at time of booking) poor or insufficient. The information time is the primary moment of the patient care pathway and it hardly conditions the quality of care. Therefore it should be specify the timing and the setting in which the patient should receive the information, to identify better the lacking moments during the information path.

The sixth question referred to a specific item of the questionnaire related to the empathy degree of all personnel involved (Graph 6). The answers were very satisfactory: the $92 \%$ (n.573) of the judged sample appreciated the staff kindness; the 6\% (n.39) considered it acceptable and only $1 \%$ (n.4) judged the staff impolite. Patients, who gave positive answers to the sixth question, gave also positive comments about the nursing care received during the pre-, intra- and post-operative period in the last open question. The $2.5 \%$ (n.16) gave an "unusual response".

The seventh question asked an opinion about the postoperative care period (Graph 7).This is a phase in which the patients experience a profound sense of disorientation and a need of security and where it is necessary a great attention by doctors and nurses. The 79\% (n.494) of the judged sample gave positive answers about doctor and nursing care received in postoperative period; the $14 \%$ (n.86) considered it acceptable, the $1 \%$ (n.5) considered it inadequate. The $6 \%$ (n.39) of

\section{How do you assessment the provided information level during the entire care pathway?}

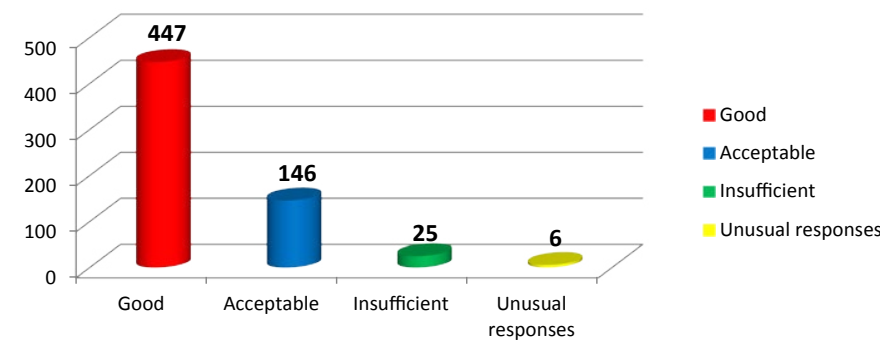

Graph 5. Question 5.

\section{How would you rate the medical staff} empathy?

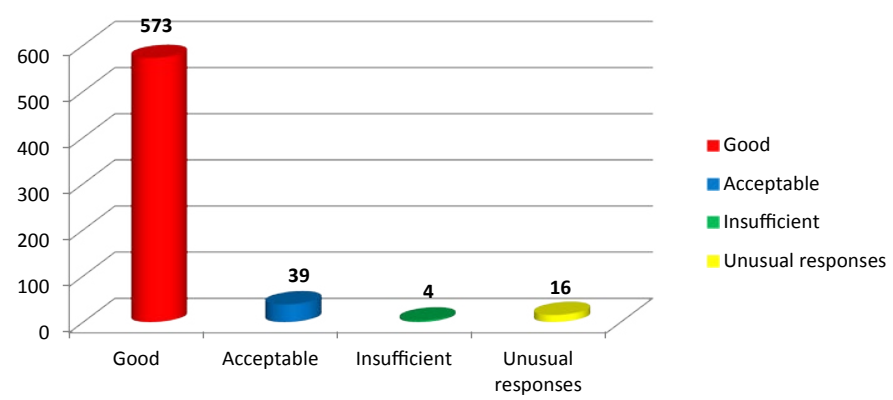

Graph 6. Question 6.

\section{How would you judge the postoperative care?}

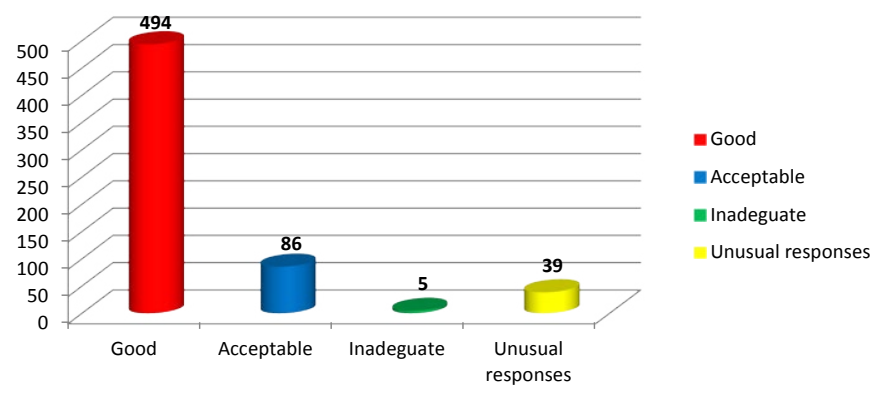

Graph 7. Question 7

\section{How would you assessment the information level at the discharge?}

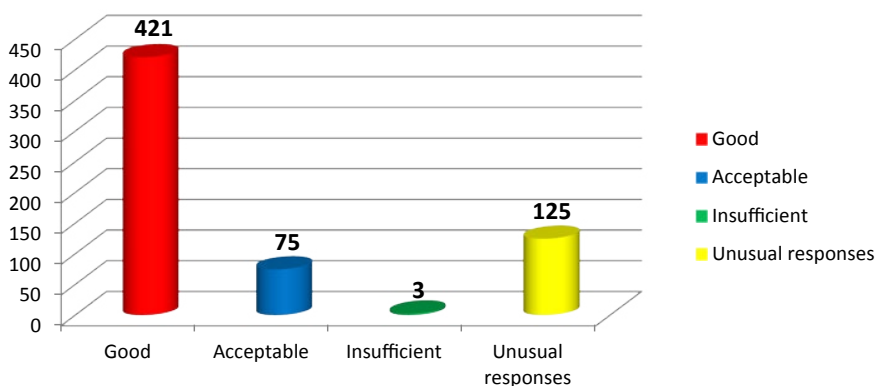

Graph 8. Question 8 .

patients gave "unusual responses" probably because many people didn't distinguish the different stages of the care pathway and they didn't understand what is the period the question was referred to.

The question number eight asked a judgment about the provided information level at the discharge time (Graph 8). The results require careful consideration. The $68 \%$ (n.421) of the judged sample considered the provided information level good; the $12 \%$ (n.75) judged it acceptable, the $0.5 \%$ (n.3) considered it insufficient, and the $20 \%$ (n.125) of patients gave "unusual responses". The high percentage of "unusual responses" highlighted the unsatisfactory level of provided information. Probably the bias origin is the administration timing of the questionnaire. The latter, in fact, was often erroneously administered before conveying the information about the discharge; therefore the patient was unable to give a relevant answer to the question.

The last multiple choice question in the questionnaire was conceived as an overall health facility and DS service assessment. A positive answer indicated a great confidence in the hospital (Graph 9). The collected data are more than satisfactory: the $95 \%$ (n.593) of the judged sample would recommend the National Cancer Institute of Naples just experienced for the future; the $2 \%$ (n.13) of patients wasn't sure, and $0.5 \%$ (n.2) didn't recommend it. The $2.5 \%$ (n.16) gave an "unusual response".

The final question is an open question in which the patient could express criticism or praise about the health facility and he could indicate any suggestions for improving care. The $84 \%$ of judged sample didn't answer to the tenth question and only $16 \%$ of patients (100 patients) gave comments. 


\section{Would you suggest National Cancer Institute of Naples to another patient?}

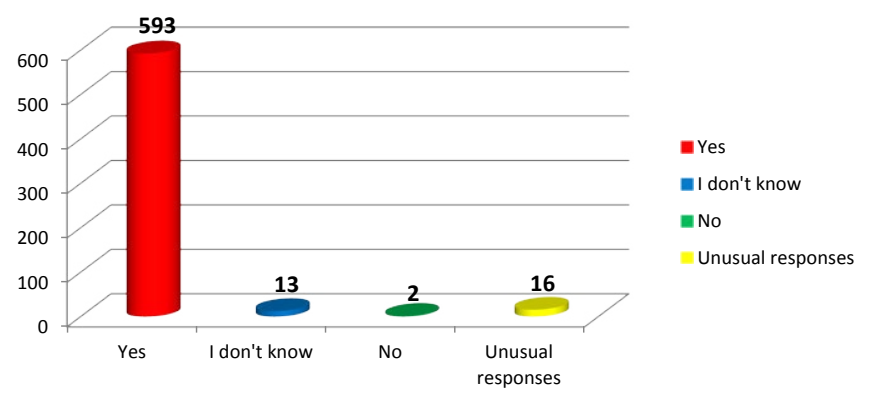

Graph 9. Question 9.

Table 3. IAS Score.

\begin{tabular}{|c|c|c|c|}
\hline Item Answers & \multicolumn{3}{|c|}{ Score } \\
\hline Item 1 & Easy (2) & Indifferent(1) & Difficult(0) \\
\hline Item 2 & Short(2) & Acceptable(1) & $\operatorname{Long}(0)$ \\
\hline Item 3 & Short(2) & Acceptable(1) & $\operatorname{Long}(0)$ \\
\hline Item 4 & $\operatorname{Good}(2)$ & Acceptable(1) & Difficult(0) \\
\hline Item 5 & $\operatorname{Good}(2)$ & Acceptable(1) & Insufficient(0) \\
\hline Item 6 & $\operatorname{Good}(2)$ & Acceptable(1) & Insufficient(0) \\
\hline Item 7 & $\operatorname{Good}(2)$ & Acceptable(1) & Inadequate(0) \\
\hline Item 8 & $\operatorname{Good}(2)$ & Acceptable(1) & Insufficient(0) \\
\hline Item 9 & Yes(2) & I don't Know(1) & No(0) \\
\hline Open answers & Thumbs Up(2) & Suggestions(1) & $\begin{array}{c}\text { Negative Comments } \\
\text { or Judgements(0) }\end{array}$ \\
\hline
\end{tabular}

IAS $\max =$ Theoretical score $(9$ items maximum score + one open answers highest score) . IAS $=$ Calculated score $(9$ items scores + one open answers score $)$

IASr $(\%)=$ IAS * 100/IAS max

The voluntary patient's comments were useful to understand patient satisfaction and/or dissatisfaction level. Unfortunately the major limitation of this method is the self-selection: only confident and decisive patients gave advices and expressed criticism or praise about the received service. It is difficult to give a relative weight to individual comments and to frame them in specific categories.

As a consequence, we formulated a specific score (Items Answers Score-IAS) assigning a value from 0 to 2 to the answers of the questionnaire with sensitive information (Table 3). The highest score was given to positive answer; the lowest score was assigned to negative judgments. We didn't consider the "unusual responses". We divided the answers to the tenth open question into three categories: the Thumbs Up to which was assigned the highest score; the Suggestions which received an average score; and the Negative Comments or Judgments to which was attributed the score 0 . If we found more than one answer, we chose only one of them.

In our study one hundred patients were more collaborative and they gave sensitive information. We divided them into two groups depending on the answer/not answer to the ten open questions. Only the $32 \%$ (n.32) of these one hundred patients answered to ten questions; the $68 \%$ (n.68) didn't answer to it. The IAS corresponded to the sum of the products of the patients number who gave sensitive information and who answered/didn't answer to the ten open question $\mathrm{X}$ the sum of the 9 items score or the sum of the 9 items + the 10 open question's answer score.

We calculated the IAS max (the perfect score) assigning a score of two to all answers. This indicated the maximum quality level and it corresponded to the sum of $\mathrm{A}+\mathrm{B}$ :

$A=68$ (the patients who gave sensitive information, but they did not answer to the tenth question) X 18 (the highest score 2 in 9 items) $=1224$

$\mathrm{B}=32$ (the patients who gave sensitive information and they answered to the tenth questions) X 20 (the highest score 2 in 10 items) $=640$.

Then, IAS $\max =\mathrm{A}+\mathrm{B}=1814$

This value (IAS max) was compared with the IAS actual (IAS), that is the IAS calculated in our study.

In our experience the IAS (corresponded to the sum of the score for the 9 , and 10 items) was:

$\mathrm{A} 1=($ sum of the score for 9 items of the 68 patients who gave sensitive information, but they did not answer to the tenth question) $=544$

$\mathrm{B} 1=$ (sum of the score for 10 items of the 32 patients who gave sensitive information and they answered to the tenth question) $=255$

Then $\mathrm{IAS}=\mathrm{A} 1+\mathrm{B} 1=799$.

The IASr, instead, is a relative score that expresses the percentage of IAS compared to reference score (IAS max). In our study IASr $=44.05 \%$.

The latter data showed a failure of the study because the percentage of provided service was less than $50 \%$ of the theoretical value. In light of this consideration, it is worth saying that the difference between the theoretical score and the calculated score hadn't absolute meaning. The IAS max indicated the highest expected quality level; its meaning was to provide a perfect service in all its components however the PS assessment was made at different times and in different settings. Several biases would be present in the score calculation:

i) Many variables conditioned the score (e.g. the canceled answers number, education level, etc)

ii) The questions in the questionnaire were referred to different provided quality aspects

iii) The comments should have a relative weight greater than the answers to the others questions; they were the true expression of what the patients perceived. Their analysis is a complex process and requires long times, the choice of a single comment among several given comments simplified it. In the case of opposites comments (one positive and one or more negative) only negative judgment conditioned the score. In our study only two patients gave more than one opinion and these comments had the same value (one positive and one negative).

iv) The judged sample. The patients in the groups B and B1were more cooperative and they gave more relevant answers to the questions then the patients in the groups $\mathrm{A}$ and $\mathrm{A} 1$. The non-responders percentage was about $68 \%$ in the groups B and B1 while it was $84 \%$ in the groups A and A1.

The combination between the numeric values (IAS), obtained by cooperative patients, and the answers results to the items represents a more objective analysis. This dual control study would have an overall quality estimate and it should highlight the lacking moments in the entire care pathway.

\section{Discussion}

A patient who needs to healthcare has a basic need; he is particular 
Cascella M (2015) Patient's perspective during surgical and/or diagnostic procedures performed in day surgery in breast cancer patient. Psychological implications and care pathway quality assessment with a specific questionnaire and a quality score

fragile and he has a state of great anxiety. This is the reason why, recently, special attention has been given to health service aspects which conditioned the medical care quality: Mitchell [12] focused the attention to the anxiety management in DS; Oudhoff et al. $[13,14]$ studied the patient psychological and social effects of the waiting list time and the need to the reduction of the waiting lists length to improve the health care service.

The concept of quality is a relative concept, it depends by patient point of view; the features of the provided service are due to patient expectations and needs. For Zeithaml et al. [15] quality is the degree of discrepancy among patient expectations and wishes and his perception about the received service. Quality is given by the difference between what the health facility is able to offer and what the patient expects to be offer to him.

In order to understand if the provided service has a good quality it is necessary to assess several elements which together define the quality. This evaluation is possible during three moments in which the service occurs:

- the conditions in which it occurs (the structure)

- the manner in which it occurs (the process)

- the result obtained (the results)

The patient's perceived experience and their satisfaction or dissatisfaction degree are different during these three moments so that the PS is different and it depends by the level of the service provided quality during the three different times [16].

The PS degree shows an overall picture of the entire care pathway: the hospital features, the comfort degree, the technical management, the doctors-patient relationship. Its analysis is useful to improve healthcare quality and to modify pathway lacking moments. Cognitive, emotional and socio-cultural factors influence PS and it is hardly evaluate with psychometric tests. Chanthong et al. [17] said that actually there aren't tests to assess certainly patient satisfaction degree. A high PS level indicated a medical staff success to satisfy patient expectations. Patient's assessment about the received service depends by objective and subjective factors, and it is subject to what is actually given to him and what he expects by the service.

These considerations are more relevant in DS especially in cancer patient. These patients are more fragile than non-cancer patients and it should be pay special attention to the emotional components during the reception, at the surgery time and in the postoperative period and to the improving of PS in this context. The overall service quality provided depends by the received service quality at different times of DS pathway and it is obtained by the results of the questionnaire.

All the health services have a technical (doctors and nurses competences etc.) and a functional (human and relational dimension, comfort) aspect. The patient is unable to give judgments about the first aspect but he knows the functional care elements that should never be overlooked.

It is difficult to structure a PS questionnaire. It must be composed by easy to understand and relevant questions thanks to which it is possible to identify how much the singles pathway lacking moments influence the patient discomfort.

The self-assessment questionnaires used by the DS of the National Cancer Institute of Naples are an efficient questionnaire but it needs of a revision in his structure. In fact the results section above demonstrated that the patients misunderstood the meaning of some questions like the first, the second and the eighth question. In order to obtain the real patient satisfaction degree, the questionnaire should be withdrawn by the staff at the time discharge home or it should be mailed at a later time. However in the latter case large amount of the questionnaire can be lost.

In the tenth questions the patients gave positive judgments to the provided care service especially they appreciated the Assistants Hospital Volunteers staff and the nursing staff knowledge and availability.

About the $30 \%$ of the criticism was referred to the health facility especially to the hotel service ("I express a positive judgments but the hospital requires a modernization"). Others criticisms were related to the too long waiting times for surgery or to the waiting time in the day of surgery, etc.

\section{Conclusion}

Actually all health facilities needs to the perceived quality assessment questionnaires particularly when cancer diagnosis procedures are performed in DS. Despite the surgery security, the quality levels involve all the care pathway aspects with a special attention to the emotional components in cancer patient or in patients subjected to the surgical and diagnostic procedure aimed at confirmation of a suspected neoplasm.

Medical staff should have an appropriate knowledge; they must provide the required service giving an efficient psychological support to the patient [18].

The questionnaire proposed by the National Cancer Institute of Naples was composed by clear questions investigating all the moments in the DS care pathway.

The results of the analysis of data, originated by the comparison between IAS and the answers to the singles items, showed that the questionnaire had a good effectiveness. In fact, it assessed simultaneously different aspects of DS PS. In addition, it was understood to the majority of patients thank to the questions clarity.

\section{References}

1. (1992) Guidelines for Day Case Surgery. The Royal College of Surgeons of England RCSENG, Professional Standards and Regulation.

2. Association of Anaesthetists of Great Britain and Ireland; British Association of Day Surgery (2011) Day case and short stay surgery: 2. Anaesthesia 66: 417-434. [Crossref]

3. Bryson GL, Chung F, Cox RG, Crowe MJ, Fuller J, et al. (2004) Canadian Ambulatory Anesthesia Research Education group. Patient selection in ambulatory anesthesia - an evidence-based review: part I. Can J Anaesth 51: 782-794.

4. Bryson GL, Chung F, Finegan BA, Friedman Z, Miller DR, van Vlymen J, et al. (2004) Canadian Ambulatory Anesthesia Research Education group. Patient selection in ambulatory anesthesia - an evidence-based review: part II. Can J Anaesth 51: 768-781.

5. Bettelli G (2009) High risk patients in day surgery. Minerva Anestesiol 75: 259-268 [Crossref]

6. Bellani ML (2008) Psychological aspects in day-case surgery. Int J Surg 6 Suppl 1: S44-S46. [Crossref]

7. Li JT (2006) The quality of caring. Mayo Clin Proc 81: 294-296. [Crossref]

8. Griffin IS, Fentiman M (2002) 17. Psychosocial problems following a diagnosis of breast cancer. Int J Clin Pract 56: 672-675. [Crossref]

9. Mitchell M (2012) Influence of gender and anaesthesia type on day surgery anxiety. $J$ Adv Nurs 68: 1014-1025. [Crossref]

10. Harding MM (2014) Incidence of distress and associated factors in women undergoing breast diagnostic evaluation. West J Nurs Res 36: 475-494. [Crossref] 
Cascella M (2015) Patient's perspective during surgical and/or diagnostic procedures performed in day surgery in breast cancer patient. Psychological implications and care pathway quality assessment with a specific questionnaire and a quality score

11. Margolese RG, Lasry JC (2000) Ambulatory surgery for breast cancer patients. Ann Surg Oncol 7: 181-187. [Crossref]

12. Mitchell M (2000) Anxiety management: a distinct nursing role in day surgery. Ambul Surg 8: 119-127. [Crossref]

13. Oudhoff JP, Timmermans DR, Knol DL, Bijnen AB, van der Wal G (2007) Waiting for elective general surgery: impact on health related quality of life and psychosocial consequences. BMC Public Health 7: 164. [Crossref]

14. Oudhoff JP, Timmermans DR, Bijnen AB, van der Wal G (2004) Waiting for elective general surgery: physical, psychological and social consequences. ANZ J Surg 74: 361-367. [Crossref]
15. Zeithaml VA, Parasuraman A, Berry LL (1990) Delivery quality service: balancing customer perceptions and expectations. New York: Free Press.

16. Gasquet I, Villeminot S, Estaquio C, Durieux P, Ravaud P, et al. (2004) Construction of a questionnaire measuring outpatients' opinion of quality of hospital consultation departments. Health Qual Life Outcomes 2: 43. [Crossref]

17. Chanthong P, Abrishami A, Wong J, Herrera F, Chung F (2009) Systematic review of questionnaires measuring patient satisfaction in ambulatory anesthesia. Anesthesiology 110: 1061-1067. [Crossref]

18. Gilmartin J, Wright K (2007) The nurse's role in day surgery: a literature review. Int Nurs Rev 54: 183-190. [Crossref]

Copyright: $(02015$ Cascella M. This is an open-access article distributed under the terms of the Creative Commons Attribution License, which permits unrestricted use, distribution, and reproduction in any medium, provided the original author and source are credited. 